next rise of temperature was on Jan. 16th, 1883, when it reached $103^{\circ}$ at 9 P.M., the pulse being then only 76 ; but at 10 and 11 I'M. he had severe attacks of dyspnoea, the face and lips becoming duslky. Before this time the sensibility had been quite restored in his legs. After this, for many months his temperature was never $r$ re than half a degree above normal, more commonly half a uegree below it; but on May 20 th it rose to $109.2^{\circ}$, he was sweating profusely, and his face was dusky, but his pulse only 80 , and respiration 22. Half an hour afterwards, on his bowels being freely relieved, he said at once that he felt much better, and his temperature was found to have fallen to $97.8^{\circ}$-i.e., $11.2^{\circ}$ in three-quarters of an hour. Again, on June 19th, he complained of abdominal pain and discomfort, and his temperature rose rapidly to $110^{\circ} 4^{\circ}$. Immediately after his bowels were relieved, and then the temperature fell $11 \cdot 6^{\circ}$ within half an hour, and continued falling till it reached $98^{\circ}$. After this only one very great rise took place -namely, in March, 1884; on other days the temperature varied only from $98^{\circ}$ to a little above normal. No deformity occurred in his spine, no "contracture" or persistent rigidity in his legs, nor more wasting of their muscles than could be accounted for by disuse. His general nutrition was well maintained. The incontinence of urine and fæces was persistent, but he was conscious of their evacuation, and could utilise a urinal.

In May, 1884, he was removed to the infirmary of the union workhouse, where he remains (May, 1885) in much the same state, keeping his bed. We have no thermal reports from this infirmary, except that on April 30th, his bowels being confined, his temperature was found to be $104^{\circ}$, and when they were relieved became normal.

Remarks.-The case shows that enormously high temperatures of the body are not necessarily dangerous to life, if they be transient, and the occasion on which a temperature of $109^{\circ}$ was accompanied by a pulse of 80 and respiration 22 in a minute shows that an excessive heat of the blood does not per se much accelerate the heart's action or the respiration. The mere facts of the case seem worth recording, but its chief interest would be in the causation of the high temperature, if this could be explained satisfactorily. It seems worth while to make the attempt. It appears that the risings of temperature were due to increased production of heat, not to checks on its loss. That a chief condition of its production must have been the lesion of the spinal cord. That, having regard to recorded ${ }^{2}$ cases of injuries of the cervical cord, and of the effects of its section in animals, we may infer that the seat of the lesion was of importance. But it is a point of interest that the lesion in this case was lower, being not in the cervical but in the dorsal cord at or near the origin of the second dorsal nerve. That the increased production of heat took place not equably in all parts of the body, but chiefly, if not solely, in parts physiologically below this point in the dorsal cord. There is evidence of this in the axilla and rectum temperatures being much higher than that of the mouth. That these parts include not only a vast majority of the muscular masses, but also the liver and other abdominal organs to which the splanchnic nerves are distributed-i.e., the two chief seats of heat production. But as the lesion of the cord was permanent, and the risings of temperature only occasional and so strangely transient, the chronic lesion could not have been the sole cause, however essential as a condition. This occasioning cause must have been of a transient kind, for its immediate effects were fleeting, and it left little, when it left any, after effects. What was it? The obvious suggestion of a renewal of inflammation, or even mere congestion, of the cord seems inconsistent with phenomena so transient. No such phenomena had been observed in the first stage of the disease. Of other hypotheses there seems only one to which there are not conclusive objections. This is suggested by a symptom by which the strange risings of temperature were preceded or accompanied-the symptom of irritated bowels, irritated by retained fæces or by purgatives. That some relation did exist between the intestinal irritation and the abnormal production of heat is evidenced both by their close sequence or concurrence in time, and by the equally striking fact of the rapid falls from high to low temperatures immediately on the bowels being relieved of the irritation. It is no doubt conceivable that the bowel irritation and rise of temperature were both effects of the same cause. But the irritation was on most occasions distinctly observed to precede the rise of temperature, and its subsidence to be followed by the fall. The sequence suggests that the former may have been the cause of the latter. IIow it might be so is not obvious, yet is not wholly beyond explanation. It is conceivable that reflex action through the cord might stimulate to increased metabolism. But, besides the want of evidence that such stimulation of the cord can raise the temperature, there is the objection that stimulation of a splanchnic nerve would constrict the arteries in its vascular area, and thereby check the production of heat. The effect might, however, be produced by inhibitory influences. The condition of the patient, with his spinal lesion, was like that of an animal which has recovered from the first effects of experimental section of its spinal cord. At the point of lesion was an impediment to vaso-constrictor impulses proceeding downwards from medulla and cervical cord; and the great thermogenic areas below the lesion were in a state abnormally favourable for the dilatation of their minute arteries, and thereby for increasing their supply of blood and consequently the metabolism of their tissues. Intestinal irritation would be directly inhibitory of local tone in the vascular area of the bowels, and would be the more efficient because the tone was already enfeebled as a consequence of the spinal lesion. And, besides this, there is evidence that the over-production of heat took place, not in the abdominal organs only, but in all parts of the body physiologically below the spinal lesion. This more general effect might be the result of afferent impulses, through the splanchnic nerves, inhibiting the feeble vaso-tonic influence proceeding from the small portion of the cord between the origin of those nerves and the lesion above them, and inhibiting likewise any enfeebled tonic impulses which may have proceeded from the cervical cord and medulla, or from the sympathetic ganglia. Vasodilatation would follow on these inhibitions, and rasodilatation in the abdominal viscera and greater part of the muscles would largely augment the production of heat. I am aware that this explanation falls far short of a demonstration. The causation of the phenomena is obscure; but if the suggested explanation be well founded, our view would be enlarged as to the agency of afferent nerrous impulses in the thermo-taxic mechanism, and it would be more restricted than at present as to the agency of the supposed thermal centre in the medulla. It would suggest that the controlling function of this centre is confined to checking the production of heat; and that among the chief causes of its production are not only impressions of cold on the skin, but also impressions on the inner surface of the intestinal canal. Cambridge.

\section{CLINICAL NOTES ON THE TARSAL TUMOUR.}

\section{BY J. VOSE SOLOMON, F.R.C.S.}

PROFESSOR OF OPHTHALMIC SURGERY IT OURFY'S COLLEGE, AYR SEYIOR SURGEON OF THE EYE HOSPITAL, BIRMINGHAM.

THe tarsus is frequently the seat of a well-defined tumour, of the size of a boiled green pea, which has a tendency to soften and discharge itself by ulceration through the cartilage on its conjunctival surface. It is a disease of the Meibomian follicles, from which no age is exempt, though it is more frequent in the interval of adolescence and the middle period of life, and oftener among women than men. ${ }^{1}$ I have seen the tumour in an infant in arms, and occasionally in a man well advanced towards the patriarchal term of life. It varies in size and number. I operated upon one as large as half a cob-nut, which almost completely covered the upper eyelid, and caused acute inflammation of the integument covering it. The disease is often solitary; but I have seen the upper eyelid studded with little tumours of the size of partridge-shots, and in this instance they had excited considerable distress and conjunctival inflammation. In the upper lid the tumour is more often situated near its centre than in the lower, where the extremities of the cartilage appear to have a greater predisposition to the disease; and it is here also that the morbid process extends occasionally into the free margin of the tarsus. In cutting into a tarsal tumour, pus sometimes escapes from its centre, no indication

In eighty-nine cases registered at the Birmingham Fye Hospital, 65 per cent. were females and 33 per cent. males. I am indebted to Mr. Ensor, our house surgeon, for these figures. 
of its presence being afforded by the colour of its integument, which retains in many cases a normal appearance; whereas in others the growth of the tumour not infrequently excites inflammation of the skin covering it, and the underlying cellular tissue may become the seat of an abscess. The evacuation of pus by incision from either of these situations is not followed by reduction of the inflammatory state, the tumour apparently acting as a foreign body, for on its removal all irritation soon subsides. In a few cases complaint is made that the vision is dim, which disappears on removal of the substance; in some there is pain with a sense of weight, on account of which surgical aid is sought. The tarsal tumour sometimes disappears by absorption, independently of treatment of any kind. Its tendency is to soften, evidence of which is afforded by the appearance of a dark mark of irregular outline on the conjunctival side of the swelling; and if not interfered with, this part ulcerates, the jelly-like contents escape, and a rather hard buttonshaped granulation results, which by. friction on the eyeball may occasion much pain and inconvenience.

In a case recently under my care at the Eye Hospital the tumour, which had not softened, bled when sliced as freely as if it had been a nævus. If the removal of the tumour is decided upon prior to softening having taken place, the whole of the disease should be excised, or the cure will not be complete, and reproduction be almost certain. And where the growth has invaded the free border of the tarsus it must be removed by the knife, care being taken that the notch in the cartilage does not implicate the skin. Large tumours in a state of inflammation involving the integument are most conveniently treated by excision externally. Where softening has been evident, I have sometimes trephined the conjunctival aspect of the growth, but it is more painful than a crucial incision. The application of solid nitrate of silver to the walls of the emptied cyst $I$ hold to be a barbarism altogether unnecessary, and often occasioning much suffering and loss of time to the patient. Birmingham.

\section{NECROSIS OF LUMBAR VERTEBRA OF OBSCURE ORIGIN; RAPIDLY FATAL RESULT.}

Bx JAMES Y. TOTHERICK, M.D., HOYORARY PHYSICIAY TO THE WOLVERHAMPTON GEYERAL HOSPITAL.

ON Sunday, March 22nd, I was sent for by Mr. Cooke of Tettenhall, to see with him a young gentleman, aged fifteen, who had been complaining for some days of symptoms which were difficult to understand. On the Wednesday preceding he had called on Mr. Cooke, stating that he had a pain in the stomach, but not looking particularly ill, and doing his school work as usual. On the next day, not feeling so well, he kept his bed, and Mr. Cooke saw him. He then complained of severe pain in the back, which was tender to the touch, and in the abdomen, which was distended. His temperature was raised and his pulse quickened. He was very closely questioned as to whether he had had any accident or injury, but steadily maintained that he had not. Nor could it be made out that he had eaten anything injurious, the only questionable thing having been some preserved plum-pudding the previous Sunday. These symptoms - the abdominal distension and pain and the tenderness in the dorsal region-continued and increased, with a temperature which had risen to $102 \cdot 5^{\circ}$, and a pulse of 100, until the Sunday afternoon when I saw him. At this time the patient was restless, flushed, moaning with pain, impatient of being touched anywhere, but shouted on any attempt being made to handle the greatly distended abdomen, or the back in the lower dorsal region, and for about three inches on each side of the spinal column. Temperature $102^{\circ}$; pulse 100 ; respiration 22 . Six leeches had been applied to the back, and had given some temporary relief, but it was evident that the general progress of the case was rapidly down-hill. The bowels had been opened both by castor oil and enema, and there had only been a little sickness, once. The urine was normal. Six leeches were again ordered, and opium was given to relieve the pain and the distension, which were thought to be due to peritonitis. Next day the condition was much the same; the symptoms perhaps somewhat relieved, but the general condition not improved. In view of what we considered to be the condition of peritonitis without any obvious cause, we thought that a surgical consultation was advisable, and the same day Mr. Vincent Jackson saw the case with us. He, however, decliued to interfere by any surgical exploration, not considering such a step justifiable by the history or state of the case. The temperature had risen to $102.5^{\circ}$. The patieg was somewhat delirious, loudly complaining if handled \& moved in any way. For the next two days there was no material alteration in the symptoms. The temperature had once risen to $103^{\circ}$, but on the Thursday had come down to $100^{\circ}$, and the tympanites and most of the tenderness over the abdomen had disappeared. The bowels opened naturally. Urine had to be drawn off by catheter night and morning. About this time appeared some swelling and tenderness in the left elbow, and the next day a red and painful swelling in the first two knuckles of the left hand, and in each place there was fluctuation. It was now evident that we had to do with a case resembling pyæmia, but what had given rise to it was not manifest. The boy was, however, put upon pretty frequent five-grain doses of quinine and some stimulants, with apparent temporary relief; but the temperature again rising very rapidly, the cold pack was employed, with the effect of keeping the temperature quite manageable. The stupor, however, increased, and early on the Sunday morning he died quietly.

Autopsy, forty-four hours after death.-Cadaveric rigidity only in lower limbs. Red staining and leech marks on back. Abdomen distended and of a blue colour. Head: Cerebral membranes healthy; veins fuller on right side than left; brain itself normal. Thorax : Left pleural cavity contained seven ounces of turbid serum; left pleura covered with a thick layer of recent plastic lymph making the lung adherent; right lung also adherent but less markedly so pericardium healthy; partly decolourised clots in both ventricles; heart itself healthy. In the upper lobe of the left lung were numerous small white lobules, some of which contained liquid pus. The lower lobe was collapsed but floated in water. The right lung was crepitant in the upper lobe, but on the free edge were four wedge-shaped consolidated patches dark in colour, and in these patches were small areas of softening. The rest of the lung substance was emphysematous in the upper lobe. In the middle lobe were similar patches of consolidation with areas of softening. The lower lobe was almost solid, yellowish soft patches being scattered through its substance, some purulent and some apparently caseating. Spleen healthy. Liver: Pale, soft, and large in one spot was a collection of pus about the size of a shot. Kidneys: Soft in texture, of the usual size, and rather dark in colour. Intestines and peritoneum: Both healthy, with some post-mortem staining. In the first and second carpo-phalangeal joints of the left hand were collections of thick pus, extending from inside the joints and forming abscesses outside. On opening a slight swelling of the left elbow-joint a collection of pus was also found infiltrating the joint. On turning over the body and cutting through the spinal muscles pus welled up in large quantities. The periosteum of the spinal canal was intensely inflamed, and the canal external to the cord was filled with pus, which was also infiltrated amongst the spinal muscles; but the visceral surface of the membranes of the cord was free from inflammation and healthy. On examination of the vertebræe, the body of the first lumbar was found to be quite soft and infiltrated with pus, presenting internally a marked contrast with the vertebra above it and the one below, both of which were hard and of a dark livid colour.

Remarks.-Here was a strong, robust, healthy lad, one of twins, with a good family history, suddenly and without ascertainable cause taken with a fatal illness which must apparently have begun in acute inflammation of the body of the first lumbar vertebra, and presenting in a few hours all the symptoms of acute peritonitis, without, indeed, much constipation or sickness, but with great distension and extreme tenderness. The post-mortem examination revealed a large quantity of pus, infiltrated amongst the deep muscles of the loins; but during life the most careful examinations only produced extreme pain without any feeling of fluctuation. The changes in the lungs were also quite a revelation, as during life the breathing, beyond being a little hurried, was only slightly affected, and only a few mucous râles were audible on auscultation except on the last day, when some dulness was discernible. The post-mortem examination, which was performed by Dr. Dingley of the 